INPLASY

PROTOCOL

To cite: Wang et al. Risk

Factors of Early-Onset

Neonatal Sepsis A Systematic

Review and Meta-analysis.

Inplasy protocol 202160049.

doi:

10.37766/inplasy2021.6.0049

Received: 15 June 2021

Published: 15 June 2021

Corresponding author:

Ling Zeng

zengling_1025@tmmu.edu.cn

Author Affiliation:

Critical care

Support: National Natural

Science Found.

Review Stage at time of this submission: completed but not published.

Conflicts of interest:

None declared.

\section{Risk Factors of Early-Onset Neonatal Sepsis A Systematic Review and Meta-analysis}

Wang, Q1; Gan, L2; Peng, G3; Sun, J4; Wen, D5; Huang, S6; Deng, J7; Zeng, L8.

Review question / Objective: To evaluate the possibility of reducing early-onset neonatal sepsis occurrence by identifying its risk factors, intervening to prevent the variable risk factors, and closely monitoring the immutable risk factors.

Condition being studied: Results of most previous studies that evaluated the risk factors of EOS are limited as most of these studies were single-center studies that performed partial screening for risk factors.

Eligibility criteria: The selection of articles for this study was based on the following criteria: 1) EOS diagnosed based on clear laboratory and/or clinical standards; 2) the use of multivariate regression analysis to determine risk factors; and 3) the presence of complete literature data, including OR and $95 \% \mathrm{Cl}$ of risk factors. We excluded case reports, comments, abstracts, letters, and agreements.

INPLASY registration number: This protocol was registered with the International Platform of Registered Systematic Review and Meta-Analysis Protocols (INPLASY) on 15 June 2021 and was last updated on 15 June 2021 (registration number INPLASY202160049).

\section{INTRODUCTION}

Review question / Objective: To evaluate the possibility of reducing early-onset neonatal sepsis occurrence by identifying its risk factors, intervening to prevent the variable risk factors, and closely monitoring the immutable risk factors.

Condition being studied: Results of most previous studies that evaluated the risk factors of EOS are limited as most of these studies were single-center studies that 
performed partial screening for risk factors.

\section{METHODS}

Participant or population: Neonatus.

Intervention: No.

Comparator: No.

Study designs to be included: casecontrol, cohort studies, and crosssectional studies.

Eligibility criteria: The selection of articles for this study was based on the following criteria: 1) EOS diagnosed based on clear laboratory and/or clinical standards; 2) the use of multivariate regression analysis to determine risk factors; and 3) the presence of complete literature data, including OR and $95 \% \mathrm{Cl}$ of risk factors. We excluded case reports, comments, abstracts, letters, and agreements.

Information sources: The contents of the data extraction table included: 1) basic information (the first author of each study, year of publication, country or region of publication, and EOS-associated risk factors), 2) Study data (the study sample size, number of neonates with EOS, ORs of risk factors obtained using multivariate logistic regression analysis, and the upper and lower limits of $95 \%$ Cls). If necessary, the authors (D.W. and G.P.) obtained supplementary data by contacting the authors of the published studies.

Main outcome(s): Risk factors related to the occurrence of early-onset neonatal sepsis, the pooled OR, and the $95 \% \mathrm{Cl}$ upper and lower limits were obtained to represent the correlation strength between risk factors and early-onset neonatal sepsis occurrence; the pooled 12 value was used to determine the heterogeneity of the combined results.

Quality assessment / Risk of bias analysis: Articles were selected by two independent reviewers to reduce publication bias and heterogeneity; the Newcastle-Ottawa Scale score was used to evaluate the quality of case-control and cohort studies, whereas the Agency for Healthcare Research and Quality scale score was used to evaluate the quality of cross-sectional studies.

Strategy of data synthesis: Data abstraction was performed in accordance with PRISMA guidelines. The NewcastleOttawa Scale and Agency for Healthcare Research and Quality scale scores were used to evaluate the quality of the included studies, and the fixed-effects model was used to combine the results.

Subgroup analysis: No.

Sensitivity analysis: Research results that had a significant influence on the change in 12 value before and after removal were considered as the source of heterogeneity and re-analyzed after removal.

Country(ies) involved: China.

Keywords: neonatal sepsis, risk factors.

Contributions of each author:

Author 1 - Qiang Wang.

Author 2 - Lebin Gan.

Author 3 - Guoxuan Peng.

Author 4 - Jianhui Sun.

Author 5 - Dalin Wen.

Author 6 - Siyuan Huang.

Author 7 - Jin Deng.

Author 8 - Ling Zeng. 